\section{Electronic retinal implant surgery}

RE MacLaren retina. There are many neuronal synapses separating photoreceptors from retinal ganglion cells, such as horizontal cells, bipolar cells, and amacrine cells. It is therefore reasonable to assume that secondary (Wallerian) degeneration of retinal ganglion cells will not be such an issue following photoreceptor loss as it might be following lesioning of the primary input to a typical sensory neuron.

There has been confusion from clinical observations in end-stage RP, which reveal pallor of the optic disc and attenuated inner retinal vessels - these secondary changes have been assumed to occur secondary to retinal ganglion cell loss. ${ }^{2}$ It should however be noted that the pallor seen in end-stage RP is not the same as in optic atrophy or glaucoma, where there is a white optic disc appearance consistent with visualisation of the lamina cribrosa. The optic disc in end-stage RP typically has a 'waxy' pallor and this is consistent with the preservation of axons coursing through the optic nerve. In end-stage RP, there is profound loss of photoreceptor cells and collapse of the outer nuclear layer. This brings the inner retina in direct contact to the vascular choroid to perfuse it with oxygen ordinarily destined for photoreceptors. The blood supply to the inner retina is auto-regulated and the inner retinal vessels will therefore constrict in response to the increased oxygenation derived from the choroid. ${ }^{3}$ This vasoconstriction will also affect vessels of the optic nerve head and hence a normal pink appearance becomes waxy because the ganglion cell axons are still present, but the retinal capillary bed undergoes vasoconstriction. By way of example, in endstage choroideremia where there is loss of the outer retina and choroid, the optic nerve head remains pink and well perfused, and the inner retinal vessels are generally of normal calibre. This provides indirect evidence that the waxy pallor and attenuated inner retinal vessels seen in RP relate to vascular changes and do not necessarily imply ganglion cell loss. While there will undoubtedly be some degree of ganglion cell loss in end-stage $\mathrm{RP},{ }^{4}$ the question must always be whether or not there is sufficient
Nuffield Laboratory of Ophthalmology, Department of Clinical Neurosciences, University of Oxford and Oxford Eye Hospital, Oxford University Hospitals NHS Foundation Trust and NIHR Biomedical Research Centre, Oxford, UK

Correspondence:

R MacLaren, Nuffield

Laboratory of Ophthalmology University of Oxford and Oxford Eye Hospital Department of Clinical Neuroscience Oxford University Hospitals NHS Foundation Trust and NIHR Biomedical Research Centre, Oxford OX3 9DU, UK Tel: +441865231513 . eye.ox.ac.uk Accepted: 12 October 2016 Published online: 6 January 2017

Lecture presented at the Cambridge Ophthalmological Symposium on Thursday, 8 September 2016. E-mail: enquiries@

Received: 3 October 2016 patients of working age. ${ }^{1}$ In common to the vast majority of cases of AMD and RP, loss of cells in the outer retina such as the retinal pigment epithelium (RPE) and photoreceptors may occur despite relatively good preservation of the inner 
ganglion cell survival to transmit a visual signal if we are to attempt some form of photoreceptor replacement, either by cell transplantation or with an electronic device. ${ }^{5}$

The retinal implant developed by Eberhart Zrenner et $a l^{6}$ in Tübingen in Germany has been in development for almost 20 years. The key aspect of this device that differentiates it from the epiretinal implant (Argus II), ${ }^{7}$ is its location in the subretinal space, which places it in the correct anatomical location for a pixelated image. The retinal implant also contains light-sensitive components in addition to electrodes and hence functions in a very similar manner to a photoreceptor array, with signals being transmitted directly to the residual bipolar and horizontal cells of the inner retina. The retinal implant is therefore more complex than the Argus II and has consequently taken longer to develop in clinical practice. The power supply to the retina implant originates from an induction coil behind the ear and this also requires additional surgical time for implantation. One benefit of this arrangement, however, is that there is no external camera device visible around the face, which many blind patients appreciate because they do not wish to draw attention to themselves in public. Optically, the retina implant relies on the optics of the eye to provide a focussed image on the retina and this also means that the image is scanned with the ocular saccades, which may provide a more natural interpretation of the visual scene. ${ }^{8,9}$ The implantation surgery requires the expertise of a cochlear implant surgeon and an anaesthetist who is comfortable with relatively long periods of general anaesthesia. In 2011, we gained ethics approval at the Oxford University Hospitals NHS foundation trust to implant the first generation Alpha-IMS retinal implant as part of a multicentre clinical trial to assess the retinal implant in clinical practice. The trial was part of a clinical investigation led by Retina Implant AG (RIAG) that was subsequently successful in gaining a CE Mark for the device.

\section{The Alpha-IMS retinal implant}

The light capturing part of the Alpha-IMS retinal implant consisted of a complementary metal-oxidesemiconductor (CMOS) camera-like chip with 1500 pixels, $\sim 3 \times 3 \mathrm{~mm}$ in diameter. The chip was embedded onto a polyimide band, which carried power in and signals out of the eye (Figure 1). Each pixel was $70 \times 70$ microns in diameter; comprising a light-sensitive part (photodiode), and an electrode for stimulation of the overlying retina. The photodiode is therefore like the outer segment of the photoreceptor, whereas the electrode provides the photoreceptor synapse that relays electrical signals to the inner retina. ${ }^{9}$ Both sensory and stimulatory functions are therefore incorporated into the chip, which is a main distinguishing feature compared with the Argus II device. The latter only comprises the stimulatory electrode inside the eye - an externally mounted camera captures the image. ${ }^{7}$ Considering the $3 \times 3 \mathrm{~mm}$ dimension, the retina implant spans a visual field of $\sim 12$ degrees (depending on eye size) and the pixel density could theoretically yield a best-corrected visual acuity of around $6 / 75 .^{10}$ This is
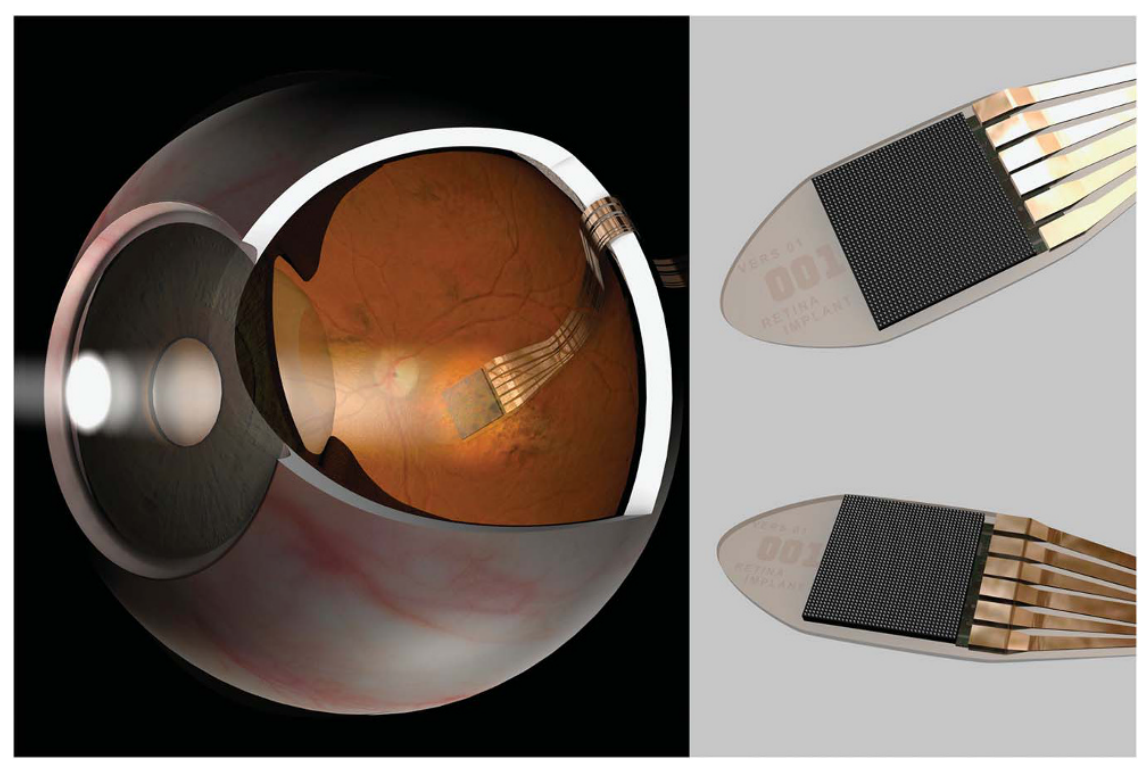

Figure 1 Diagram showing the position of the electronic retinal implant and power cable in the eye. Inset views show detail of the retina implant AG first-generation Alpha-IMS chip, which contains 1500 pixels in a $3 \times 3 \mathrm{~cm}$ array. 
however dependent on perfect contact of the individual 70 micron electrodes with overlying functional bipolar/ horizontal cells. For this reason, the chip is best placed as close to the fovea as possible because it is assumed that this region has the greatest bipolar cell density and visual cortex resolution. ${ }^{8}$ In the central macula, optical aberrations, such as coma, will also be minimised leading to a sharper image, which may further aid individual photodiode stimulation (Figure 2). In our multicentre trial, two patients came close to this maximum theoretical resolution, achieving 6/160 and 6/180 using Landholt C targets. ${ }^{10}$

In the absence of photoreceptors, there is insufficient energy from photons entering the eye to be able electrically to activate bipolar cells. Some form of amplification is needed. Nature achieves this with the photoreceptor, deriving energy from the richly oxygenated choroid and storing it through the isomerisation of 11-cis retinal molecules, which are then loaded into densely packed rhodopsin in the outer segments. For an electronic device, an alternate form of power amplification is needed and for the retinal implant this is achieved via an induction loop connection through the skin behind the ear, using a magnetic interface similar to that used to power a cochlear implant (Figure 3). This arrangement has great advantages for the patient, in that it provides a fully integrated system with no external camera or other device visible. It does, however, require a longer surgical implantation time and the expertise of a cochlear implant surgeon.

Surgical implantation starts behind the ear with the power supply, which is fitted under the periosteum into a shallow bony recess. A small incision is made in the skin overlying the zygomatic arch and a trochar is passed posteriorly under the periosteum of the temporalis muscle to bring the retinal implant and attached cable forward towards the orbit. The earth 'ground' electrode is left under the temporalis. A small notch is then drilled in the zygomatic bone into which the cable will be recessed. After a 360 degree peritomy, a surgical plane from the subtenon space is identified that passes posterior to the lacrimal gland superotemporally to link up with the zygomatic incision. The retinal implant can then be carefully manipulated into the back of the orbit, ready for intraocular implantation. A scleral trapdoor is made and a $3 \times 1 \mathrm{~mm}$ strip of choroid is exposed at the posterior base of this trapdoor, $\sim 9 \mathrm{~mm}$ posterior to the limbus. After detaching the retina from the inside, the choroidal strip is cauterised and then cut open to allow the chip to be gently pushed through the choroid into the subretinal space. Further delicate manipulations on the proximal part of the foil under direct visualisation push the chip into the optimal position distally, as close to the fovea as possible but without touching the optic nerve head. A silicone mesh on the external part of the retinal implant is then used to stitch it

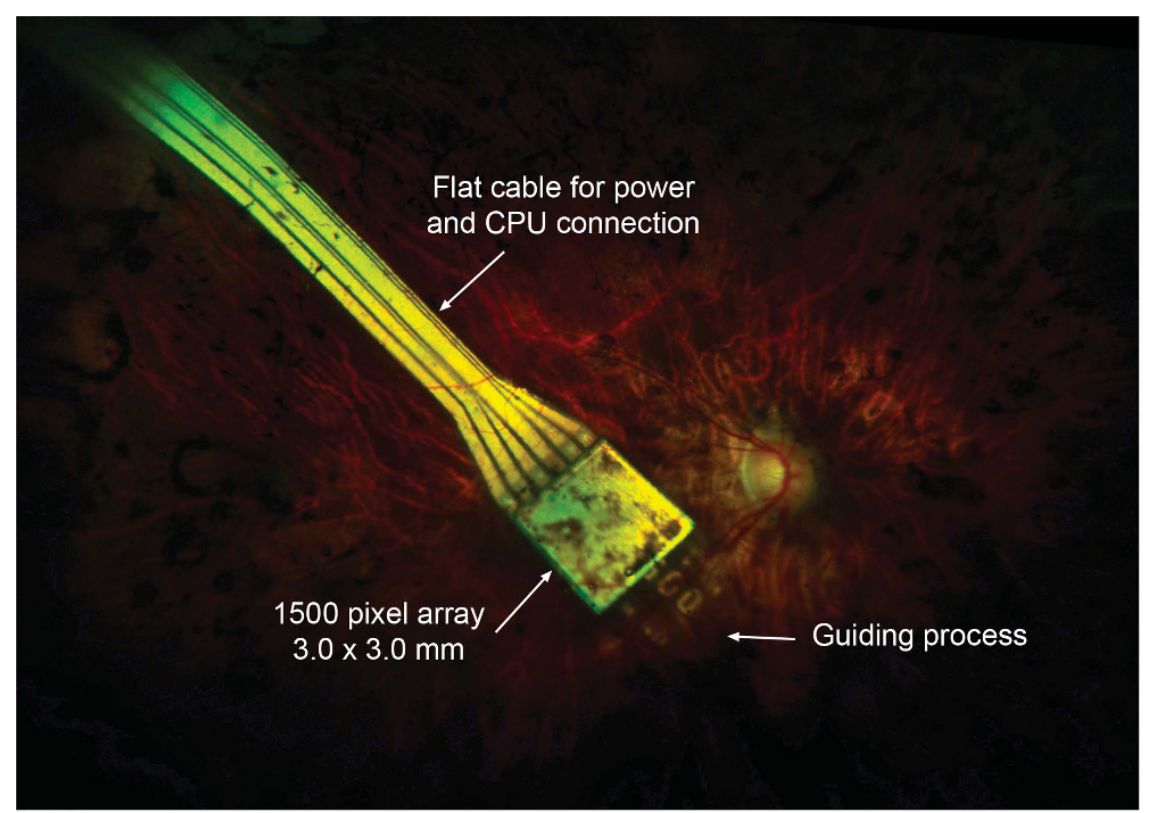

Figure 2 The position of the Alpha-IMS chip in a patient, 2 months after surgery. The guiding process helps the chip along the subretinal space when being inserted. Some mild pigmentary changes are visible in the neurosensory retina overlying the light-sensitive pixel array. Pigment migration is common in retinitis pigmentosa and very heavy pigmentation over the chip may in theory reduce light sensitivity. 
firmly to the sclera and the scleral trapdoor is sutured, thereby fixating the implant at two points. ${ }^{10} \mathrm{~A}$ firm two-point fixation is critical to prevent movement of the chip inside the eye, which might otherwise occur due to forces applied to the orbital loop during eye movements.

\section{Results of retinal implant surgery}

The trial with the first six patients using the Alpha-IMS implant in Oxford was completed at the end of 2012. Overall, the results were good, with surgery completed successfully in every patient with no significant adverse events reported. The chip restored some degree of vision to all participants, with four reporting it to be useful in activities of daily living and two reporting a little benefit (see Table 1 below). Across the whole multicentre study, 21 out of 29 participants (72\%) reached the primary endpoint of a significant improvement in activities of daily living, which included routine visually guided tasks, recognition tasks and mobility. ${ }^{10}$ The secondary end point of improved visual acuity, light perception and/or object recognition was achieved in 25 of the 29 participants $(86 \%) .{ }^{10}$

Towards the end of the 12-month study, however, the implanted devices began to fail. Explantation of most of the chips was helpful in identifying the causes of failure, which related to cable breakage and using a complex electronic device in a saline environment. ${ }^{10}$ All participants had been counselled about the fact that the longevity of the chip was untested and could only be assessed within the context of this first in man clinical trial. Nevertheless, it was a disappointment for participants and the research team in cases where the benefits of the chip had been significant.

On a positive note, however, the problems with the chip longevity have been addressed with a new 'nextgeneration' design, the Alpha-AMS, which we are currently testing in a further cohort of 6 patients. This new trial started in 2015 and is funded by an NIHR Invention for Innovation (i4i) award. It aims to assess the logistics of retinal implant surgery using the new device within the context of the NHS. At the time of writing this lecture summary, the first patient to receive the new chip is still using it after 16 months.

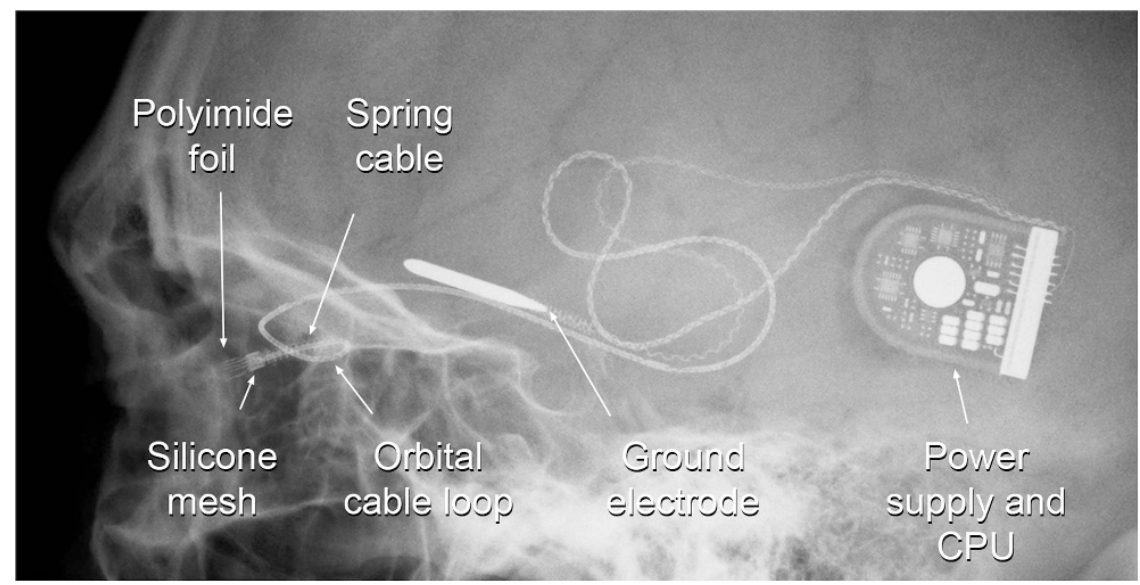

Figure 3 An X-ray showing the arrangement of the chip and polyimide foil in the eye. The silicone mesh that holds it to the sclera is also visible. The spring cable and orbital loop reduce bending forces on the electrical connections during ocular movements.

Table 1 Summary of results from Oxford patients in the Alpha-IMS trial

\begin{tabular}{ccccccc}
\hline & Light perception & Light localisation & Grating acuity $(c p d)$ & Grey levels seen & Reading clock time & Daily life experiences \\
\hline 1 & + & - & - & 3 & - & Little \\
2 & + & + & 0.33 & 5 & - & Useful \\
3 & + & - & 1 & 4 & - & Useful \\
4 & + & + & 0.33 & 4 & - & Useful \\
5 & + & + & 1 & 6 & + & Little \\
6 & + & + & + & Useful \\
\hline
\end{tabular}

Full results of the multicentre trial can be found elsewhere. ${ }^{10}$ A typical setup for testing patients can be seen in Figure 4 . To avoid any effects of guessing, subjects were tested several times without knowing if the device was switched on or off. 


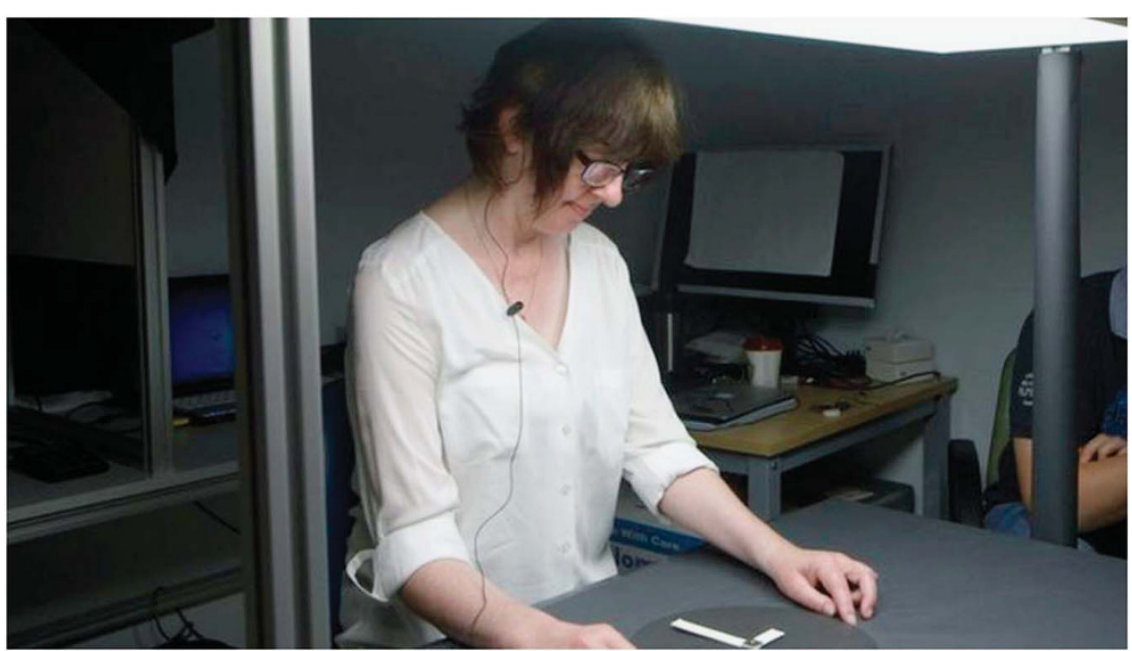

Figure 4 Participants are tested when viewing objects under controlled illumination with the implant on or off. In this case, Rhian Lewis, the first patient to receive the next generation Alpha-AMS chip, is reading the clock face. The wire connecting the power supply to the magnetic receiver behind the ear can be seen. The patient gave permission for her story to be included in a BBC television science documentary.

\section{Summary}

The electronic retinal implant is a complex device that requires complex surgery, but the results in improving vision in patients with end-stage RP have shown proof of concept and in some cases have been spectacular. There is more work to do, but this technology has now crossed the boundary from being of academic interest to becoming a useful adjunct in the management of patients with end-stage RP.

\section{Conflict of interest}

The OUH NHS Foundation Trust received clinical trial research funding from Retina Implant AG (RIAG) in order to conduct the first clinical trial as an NIHR Portfolio-adopted study. The author declares no conflict of interest.

\section{Acknowledgements}

NIHR i4i Award, NIHR Oxford Biomedical Research Centre.

\section{References}

1 Liew G, Michaelides M, Bunce C. A comparison of the causes of blindness certifications in England and Wales in working age adults (16-64 years), 1999-2000 with 2009-2010. BMJ Open 2014; 4: e004015.
2 Al Rashaed S, Khan AO, Nowilaty SR, Edward DP, Kozak I. Spectral-domain optical coherence tomography reveals prelaminar membranes in optic nerve head pallor in eyes with retinitis pigmentosa. Graefes Arch Clin Exp Ophthalmol 2016; 254: 77-81.

3 Yu DY, Cringle SJ. Retinal degeneration and local oxygen metabolism. Exp Eye Res 2005; 80: 745-751.

4 Jones BW, Pfeiffer RL, Ferrell WD, Watt CB, Marmor M, Marc RE. Retinal remodeling in human retinitis pigmentosa. Exp Eye Res 2016; 150: 149-165.

5 Cramer AO, MacLaren RE. Translating induced pluripotent stem cells from bench to bedside: application to retinal diseases. Curr Gene Ther 2013; 13: 139-151.

6 Zrenner E, Stett A, Weiss S, Aramant RB, Guenther E, Kohler K et al. Can subretinal microphotodiodes successfully replace degenerated photoreceptors? Vision Res 1999; 39: 2555-2567.

7 Humayun MSD, da Cruz JD, Dagnelie L, Sahel G, Stanga JA, Cideciyan PE et al. Study group interim results from the international trial of Second Sight's visual prosthesis. Ophthalmology 2012; 119: 779-788.

8 Stingl K, Bartz-Schmidt KU, Gekeler F, Kusnyerik A, Sachs H, Zrenner E. Functional outcome in subretinal electronic implants depends on foveal eccentricity. Invest Ophthalmol Vis Sci 2013; 54: 7658-7665.

9 Zrenner E, Bartz-Schmidt KU, Benav H, Besch D, Bruckmann A, Gabel VP et al. Subretinal electronic chips allow blind patients to read letters and combine them to words. Proc Biol Sci 2011; 278: 1489-1497.

10 Stingl K, Bartz-Schmidt KU, Besch D, Chee CK, Cottriall CL, Gekeler F et al. Subretinal Visual Implant Alpha IMS-Clinical trial interim report. Vision Res 2015; 111: 149-160. 